\title{
Pain management practices surrounding lumbar punctures in children: A survey of Canadian emergency physicians - CORRIGENDUM
}

Naveen Poonai; Victoria Brzozowski; Antonia S. Stang; Amy L. Drendel; Philippe Boisclair; Michael Miller; Stuart Harman; Samina Ali; Pediatric Emergency Research Canada (PERC)

doi: $10.1017 /$ cem.2018.382

In the original publication of this article, Pediatric Emergency Research Canada (PERC) was not listed as an author.

The authors regret this error. The original version has been updated.

\section{REFERENCE}

Poonai N, Brzozowski V, Stang AS, Drendel AL, Boisclair P, Miller M, Harman S, Ali S, Pediatric Emergency Research Canada (PERC). Pain management practices surrounding lumbar punctures in children: A survey of Canadian emergency physicians. CFEM 2018. doi:10.1017/cem.2018.382. 